\title{
Design and Development of Archives Management Information System in Primary and Secondary Schools
}

\author{
Li Nianping \\ Center for Information Development, Chongqing Municipality Yongchuan Middle School, Chongqing, China
}

Email address:

345352948@qq.com

To cite this article:

Li Nianping. Design and Development of Archives Management Information System in Primary and Secondary Schools. Science Discovery. Vol. 6, No. 5, 2018, pp. 421-428. doi: 10.11648/j.sd.20180605.27

Received: September 15, 2018; Accepted: October 18, 2018; Published: November 8, 2018

\begin{abstract}
The main way of recording school records has changed from original writing to electronic documents. A large number of electronic documents not only put forward new requirements for file management, but also bring great convenience for file digitization. With the progress of digital campus, digital archives management in primary and secondary schools is an inevitable development direction. This paper has elaborated paper archives and digital archives integrated management solution applicable to primary and secondary schools according to the requirements of GB/T 18894-2016 "Standards of electronic records filing and management", based on the development and application practice of archives management information system in Chongqing Yongchuan Middle School. The paper has emphatically elaborated the design and implementation of the archives management information system, including architecture and technology design, requirement and function design, security and confidentiality design, and business process design. Therefore, In the aspect of technology realization, In the aspect of technology realization, the idea of software development based on $\mathrm{B} / \mathrm{S}$ architecture design and layered realization is expounded. It involves the system configuration, category management, file management, box management, thematic file management and loan registration management and other major functional modules of file management.it can be used as a reference for archives digital management in primary and secondary schools.
\end{abstract}

Keywords: Primary and Secondary School, Archives Management, Information System

\section{中小学校档案管理信息系统的设计与开发}

李念平

信息发展中心, 重庆市永川中学校, 重庆, 中国

\section{邮箱}

345352948@qq.com

摘要: 学校档案信息记录的主要方式已由原来的笔写文字变成电子文档。大量电子文档既给档案管理提出了新的要求, 同时又为档案数字化带来了极大的便利。随着数字校园的推进，中小学校档案数字化管理是必然的发展方向。本文根 据GB/T 18894-2016《电子文件归档与电子档案管理规范》要求, 结合重庆市永川中学校档案管理信息系统的开发和应 用实践，阐述了适用于中小学校的纸质档案与数字化档案一体化管理整体解决方案。重点阐述了档案管理信息系统的 设计和实现，包括架构与技术设计、需求与功能设计、安全保密设计、业务流程设计。在技术实现方面阐述了基于 $\mathrm{B} / \mathrm{S}$ 架构设计和分层实现的软件开发思想。涉及系统配置、类别管理、案卷管理、案盒管理、专题档案管理和借阅登记管 理等档案管理主要的功能模块。可作为中小学校档案数字化管理的案例参考。 


\section{1. 引言}

学校档案是学校发展过程的真实记录, 是学校文化传 承和创新的宝贵财富, 是师生发展的原始依据。沿用传统 手工、纸质方式管理档案存在着查阅不便捷、原件易损伤、 无份备、管理重组难度大、统计不准确、著录不规范等问 题。随着计算机在学校的普及, 信息的记录的主要方式已 由原来的笔写文字变成电子文档。大量电子文档既给档案 管理提出了新的要求, 同时又为档案信息化带来了极大的 便利。为规范电子文档的管理, 中华人民共和国国家质量 监督检验检疫总局、中国国家标准化管理委员会于2016年 颁布国家标准 GB/T 18894-2016《电子文件归档与电子档 案管理规范》 [1], 中华人民共和国国家档案局于2017年颁 布了档案行业标准DA/T31-2017《纸质档案数字化规范》 [2]。各级档案行政和业务主管部门纷纷发文要求加强电子 文档的归档工作, 规范纸质档案数字化工作。中小学校的 档案管理如何顺应时代的发展是一个需要认真研究解决 的问题。在这样的背景下, 重庆市永川中学校根据有关档 案管理标准和文件要求, 经过技术可行性和业务可行性分 析论证后, 决定对此问题作为科研课题进行立项研究, 探 索纸质档案和电子档案一体化管理的有效方式, 提高学校 档案管理和利用的效率。在相关标准的指导下, 经过信息 技术人员和档案管理人员的共同努力, 自主开发出了中小 学校档案管理信息系统。又经过一年多来的使用和完善, 逐步形成了纸质档案和电子档案一体化管理的整体解决方 案。此套档案管理整体解决方案现已在部分中小学校推广 使用。

\section{2. 系统设计}

\section{1. 架构与技术设计}

\subsection{1. 硬件系统结构}

中小学校档案管理信息系统要求在原有档案管理硬 件的基础上配备用于档案信息化管理的基本硬件。如Web 服务器、数据库服务器各一台, 也可集中在一台服务器上。 主要用于数据的存储和系统服务。根据工作岗位和业务量 的需要配备普通的个人计算机若干。对于档案业务部门需 要配备高速扫描仪、绞边机和打印机等相关设备。硬件系 统结构如下图:

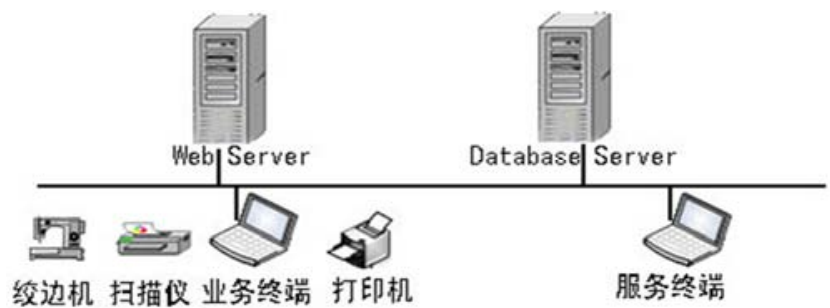

图1 硬件系统结构。

\subsection{2. 软件系统结构}

中小学校档案管理信息系统采用B/S(Browser/Server) 架构设计, 在服务器上需要安装配置IIS web服务器。客户 端通过浏览器访问, 无需要安装任务软件, 无客户端数量 限制。在.net框架下运用C\#编写支持程序。采用MS SQL Serve数据库管理系统存储和管理数据。为使架构更清晰, 代码可重用, 便于维护。按数据层, 业务层 (也有叫逻辑 层）, 表示层三层架构开发[3]。

(1) 用户界面层: 用户界面采用asp.net配合HTML 语言编写, 用户通过测览器调用显示。用户界面主要用于 收集用户输入并显示处理结果, 实现与用户交互的功能。 用户界面设计遵从用户为中心为基本原则, 设计中尽量减 少用户的认知负担, 保持界面友好, 及时提供说明和帮助, 配色统一, 图标和功能一致。用户界面主要采用CSS样式 表控制界面样式, 采用框架技术组织界面空间, 采用页面 模板技术统一界面风格, 提高设计效率。页面文件和执行 代码相互分离, 方便维护修改。页面代码经编译后封装成 DLL文件, 访客无法获得源代码。降低代码漏洞被利用的 风险。

（2）应用服务层：应用服务程序是档案管理信息系 统的核心部分, 通过 $\mathrm{C}$ \#程序设计语言编写, 运行于服务器 上, 主要通过定义以下类来实现:

数据实体类: 采用对象关系映射 $(\mathrm{O} / \mathrm{R} \mathrm{Map})$ 技术实 现, 为数据库的每个表建立相应的实体类, 如: 数据库中 的案卷表app_File对应的案卷实体类定义如下:

public partial class app_FileEntity

\{

private Int32_FileID $=0$; // 编号

private Int32_ClassID $=0$; // 类别

private Int32_QuanZongID $=0$; // 全宗号

public Int32 FileID // 编号属性

\{

set $\{$ this._FileID $=$ value; $\}$

get $\{$ return this._FileID; $\}$

\}

$\ldots$

\}

数据操作类: 封闭了实体控制、连接数据库和执行数 据库常规操作。通过操作实体类实现向数据库表中插入、 删除、修改数据, 同时为业务规则层提供数据服务。如以 下类实现案卷著录信息的增加、删除和修改。

public override Int32

RecordManage_FileInsertUpdateDelete(RecordManage_File Entity fam)

Int32 rInt $=-1$;

using $($ SqlConnection Conn $=$ GetSqlConnection( $)$ ) \{

SqlCommand $\mathrm{cmd}=$ new

SqlCommand("dbo.RecordManage_File_InsertUpdateDel 
ete", Conn);

cmd. CommandType $=$ CommandType.

StoredProcedure;

cmd. Parameters. Add("@DataTable_Action_",

SqlDbType. VarChar). Value =

fam.DataTable_Action_.ToString();

cmd.Parameters.Add("@FileID", SqlDbType.Int).Value =

fam.FileID; //编号

cmd.Parameters.Add("@ClassID",

SqlDbType.Int).Value = fam.ClassID; //类别

cmd.Parameters.Add("@QuanZongID",

SqlDbType.Int).Value = fam.QuanZongID; //全宗号

SqlDbType. VarChar). Value $=$ fam.DianZiUrl; //电子文 档

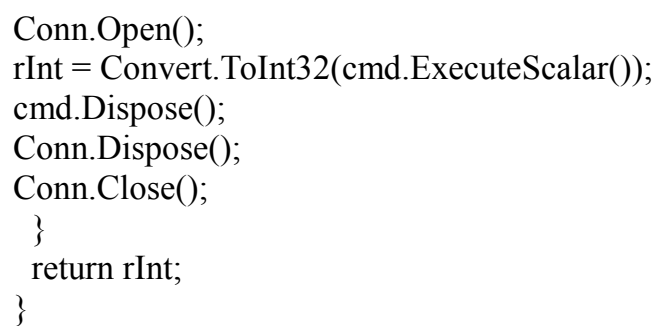

业务规则类: 各种业务规则和逻辑的实现，如帐户的 验证, 审核、多条件查询等, 为表示层提供反馈, 执行处 理过程, 使用户界面与各种功能相隔离。如以下类根据案 卷ID返回单笔记录。 public static RecordManage_FileEntity

RecordManage_FileDisp(Int32 FileID)

RecordManage FileEntity fam $=$ new

RecordManage_FileEntity();

QueryParam qp = new QueryParam();

qp.PageIndex $=1$;

qp.PageSize $=1$;

qp. Where $=$ string.Format $("$ Where $\{0\} .\{1\}=$

$\{2\}$ ","RecordManage_File","FileID",FileID);

int RecordCount $=0$;

List $<$ RecordManage_FileEntity $>1$ st $=$

RecordManage_FileList(qp, out RecordCount);

if (RecordCount $>0$ )

\{

fam $=1$ st $[0]$

\}

return fam;

\}

(3) 数据层: 选择MS SQL Server数据库, 用于数据 集中存储。数据库主要涉及用于用户管理和系统配置的基 础表、用于存储档案业务的数据表以及每个表相关的存储 过程组成。存储过程主要供程序调用, 实现对应表数据的 查询、增加、删除、修改的功能。相关表通过关键字字段 建立关联关系, 防止数据不一致, 主要关系图如图2所示 [4]。

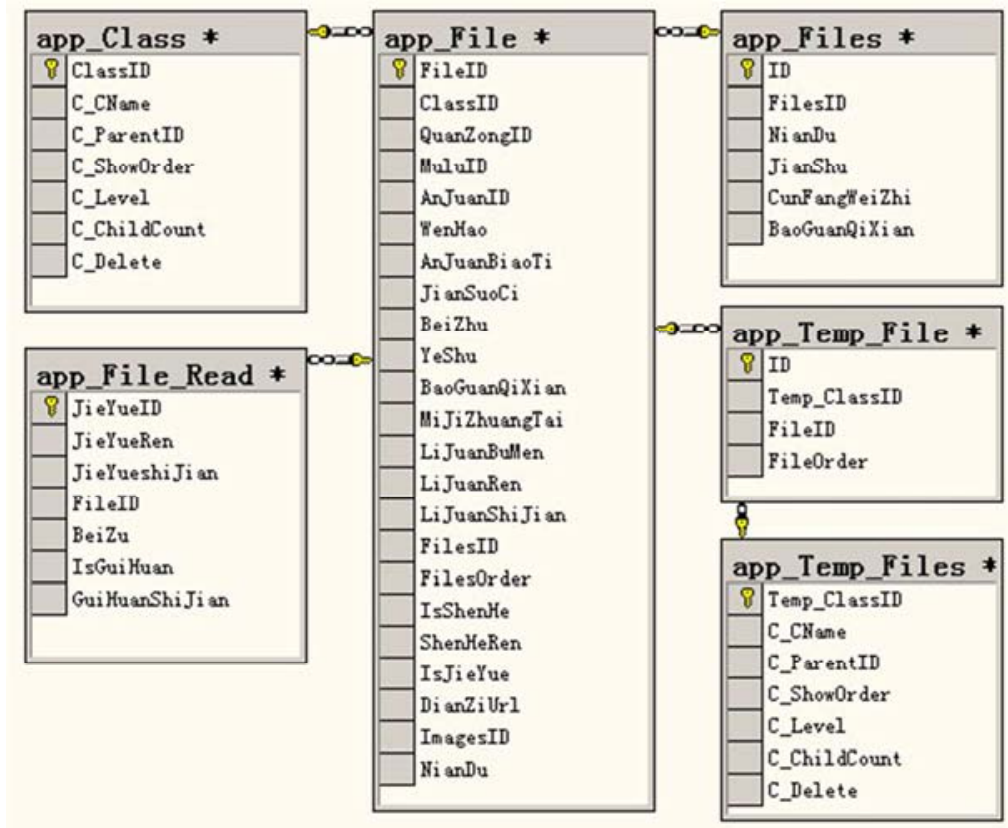

图2 数据库主要表及关系。

\section{2. 需求与功能设计}

\subsection{1. 系统配置模块}

主要实现系统登录认证, 系统参数设置, 角色权限管 理, 用户管理, 档案类别管理, 字典维护, 日志管理, 数 据备份，在线用户管理等功能。同时提供积分管理、界面 风格设置、个人信息管理等辅助功能。采用开放式设计,
便于扩充档案管理的功能模块，是整个系统的基础。用户 角色分为档案系统管理员、学校档案管理员、部门档案管 理员。档案系统管理员具备系统配置模块的管理权限。学 校档案管理员具有档案管理业务的最高权限, 负责审核部 门档案, 为案卷统一编号, 验卷归档等业务。部门档案管 理员负责立卷, 归盒。除角色设计外, 系统还设计有权限 管理。权限包括建立、删除、阅读、修改、打印、排序以 
及基础配置权限。属于不同角色的用户在系统管理员赋予 的权限范围内具有权限 [5]。系统日志包括操作日志, 安全 日志两大类型, 所有的用户操作都将记入系统日志, 确保
可追溯。基础的信息纳入字典管理, 方便维护。数据库定 时备份, 确保数据安全。以上所有的操作都可系统配置模 块具体通过菜单[系统配置]中操作, 界面如图3所示。

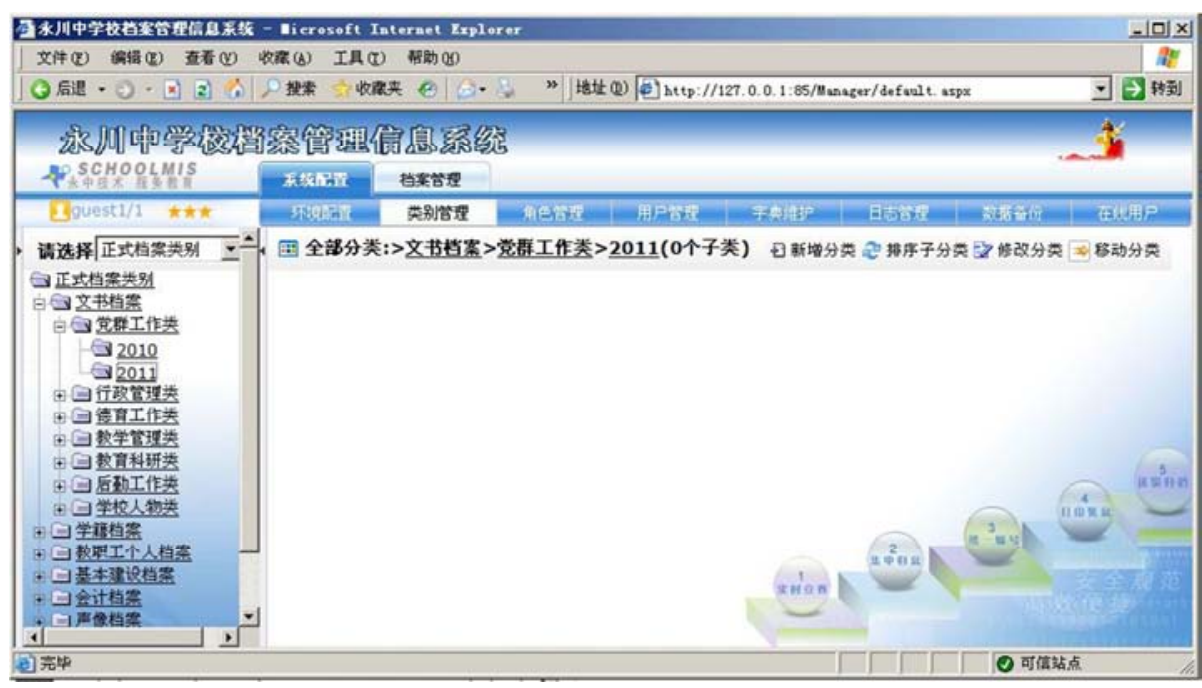

图3 案卷类别管理。

\subsection{2. 案卷类别管理模块}

主要实现正式案卷类别和专题案卷类别的建立、汶览、 排序、修改、移动、分类统计等功能。采用树型目录结构, 层次关系一目了然。本模块需要学校档案管理员权限, 具 体在[系统配置]-[类别管理中]操作。

\subsection{3. 案卷管理模块}

案卷管理模块是档案管理信息系统的核心部分, 主要 实现案卷的分类别、分期限著录编辑、查询汶览、归盒归 档, 转换标识和电子文件打印等功能。
（1）案卷的著录编辑: 包括设置了提示、默认值和 校验程序的标题、检索词、文号、备注、页数等输入类项 目; 包括类别、保管期限、电子文档等选择类项目; 包括 年度编号、统编号、全宗号、立卷人、立卷部门、立卷时 间、审核人、审核时间、文档大小、页数等由系统在相应 阶段通过程序自动生成的项目。案卷著录是档案管理的基 础性工作, 著录信息严格遵从相关规范, 配以必要属性的 验证程序, 及时提供操作要求提示, 确保著录信息全面规 范, 操作简易快捷[6]。

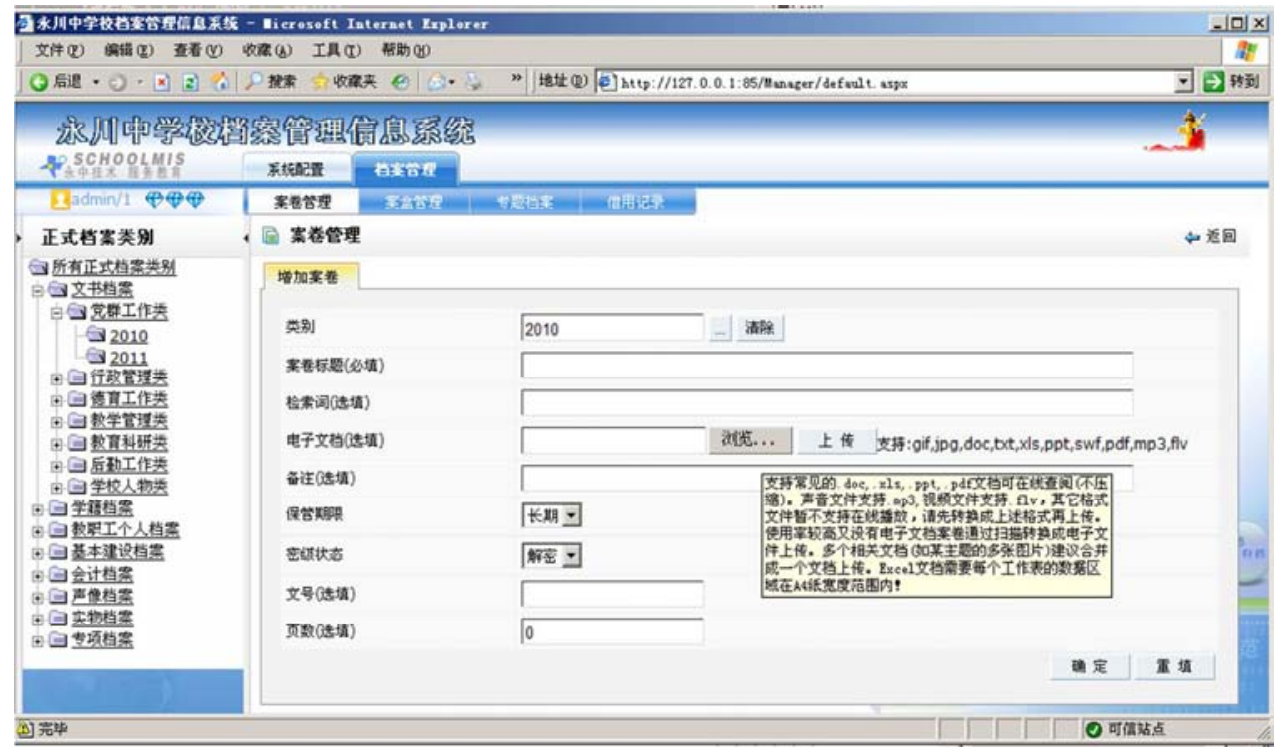

图4 案卷著录。

在案卷著录程序中设计有文件格式自动转换程序, 通 过编程实现将新建案卷上传的普通电子文档转换成标准
的PDF格式文档。案卷的著录具体在[案卷管理]-[新建案卷] 中操作, 界面如图4。 
（2）案卷的查询汶览: 案卷的查询和汶览是档案管 理信息系统常规操作和价值体现。著录的案卷可分类查询、 可自定义查询。可查项目几乎包括了案卷的所有属性, 可 根据单一属性查询亦可使用属性组合查询; 查询结果以列
表的方式显示案卷的关键信息和属性。也可进一步汶览案 卷的属性和电子文档的详细内容。凡学校档案管理员没有 审核编号的案卷, 在有权限的情况下还可进行删除、修改、 逻辑归盒等调整操作。界面如图5所示。

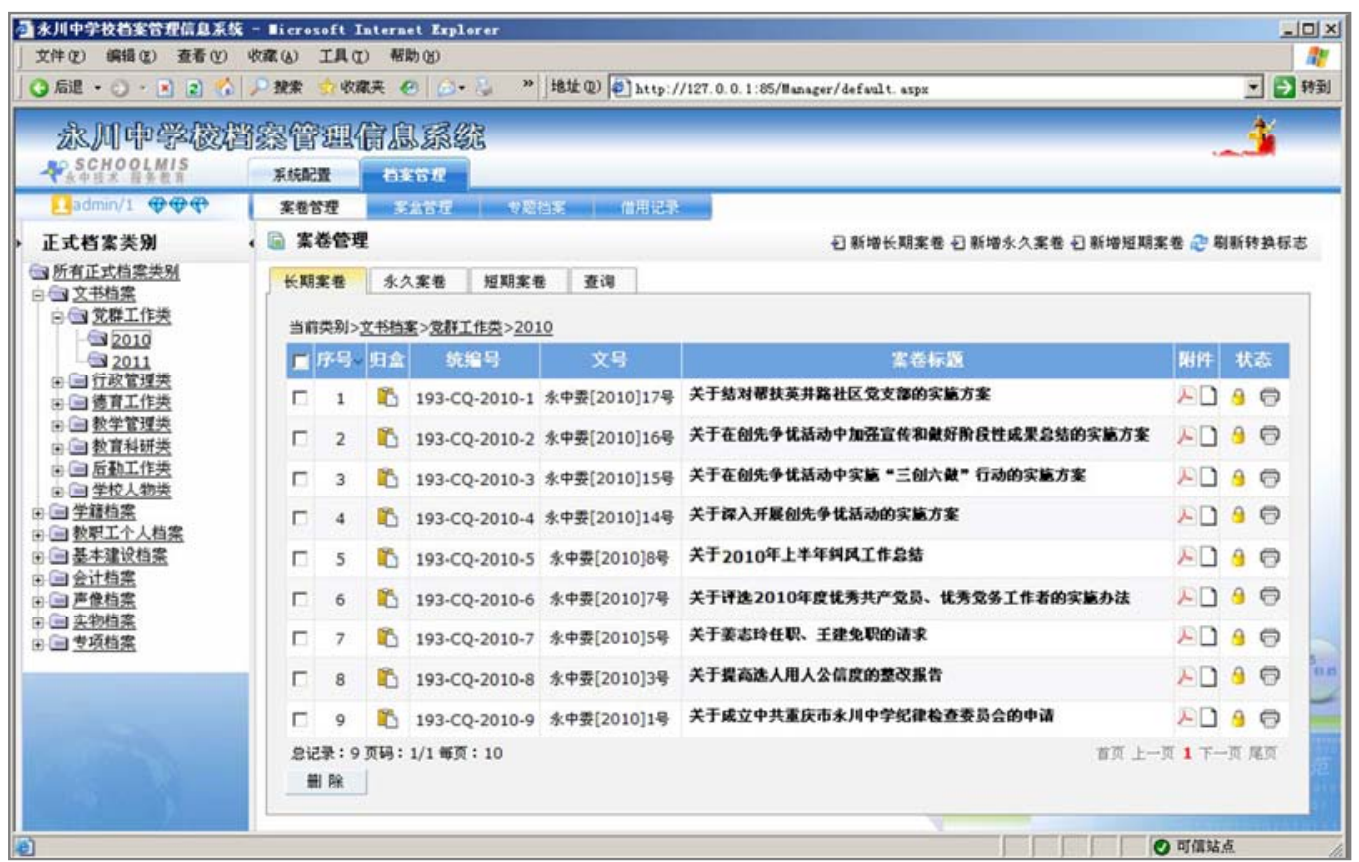

图5 案卷汶览查询。

（3）其它功能：对于已经审核归档的案卷, 需要进 行统一规范的标识。案卷标识由案卷编号、编号条形码、 类别、归档位编成组成。通过编程按系统设置的规则进行 标识, 其中案卷编号是案卷唯一性的编号。在档案管理信 息系统中支持电子文档的在线浏览和打印。也支持对案卷
属性、案卷标识的单独显示和打印。编号完成后再才从系 统中打印纸质案卷, 以实现电子案卷和纸质案卷的编号一 致, 方便后期管理。一般的案卷查阅通过在线方式进行, 为保证档案信息的安全, 档案查阅功能纳入权限管理。如 图6所示。

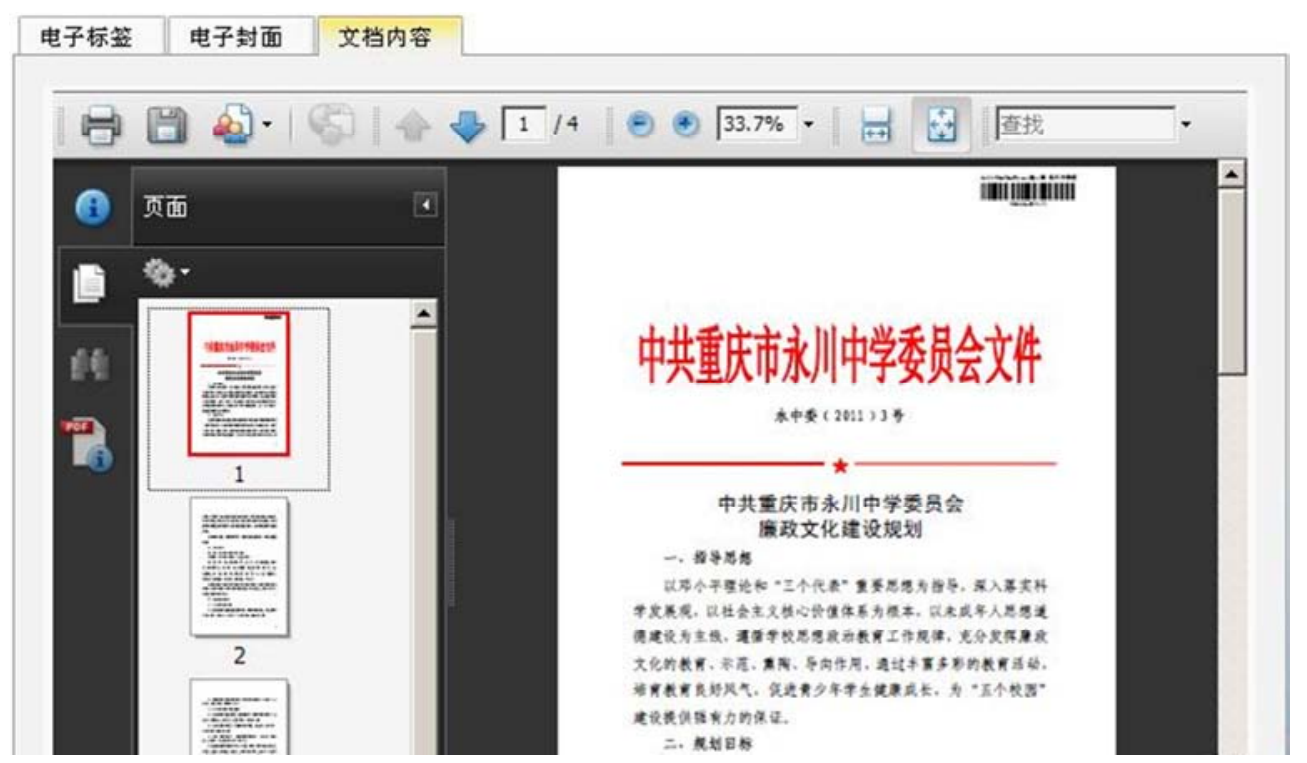

图6 案卷在线浏览打印。

\subsection{4. 案盒管理模块}

案盒管理主要实现案盒的新建, 编辑, 删除, 排序、 查询、编号、归位和盒内案卷查看、排序等功能。设计有
永久、长期、短期三个类别。界面如图7所示。案盒管理 流程设计如下: 首先是部门管理员根据需要建立相关案盒, 并将相应案卷进行预归盒处理。其次, 由学校档案管理员 
进行必要的修改、排序, 通过设计的程序对达到存档条件 的案盒按系统配置中的编号规则自动为案盒编号。第三是 根据纸质案盒将要存放的位置进行虚拟入室归位[7]。第四 是将所有案盒按规则进行统一连续编号, 然后对案盒中的 案卷进行统一连续编号, 保证每一案盒和每一案卷都有唯
一的规范编号。统一编号的案盒和案卷为正式归档, 将在 案卷对应的PDF格式的电子文档首页页眉处正式写上标 识水印。界面如图6所示。

案盒部分设计了打印功能, 可在线预览并打印案盒、 封面、卷内目录和盒脊, 使用纸质案盒统一规范。

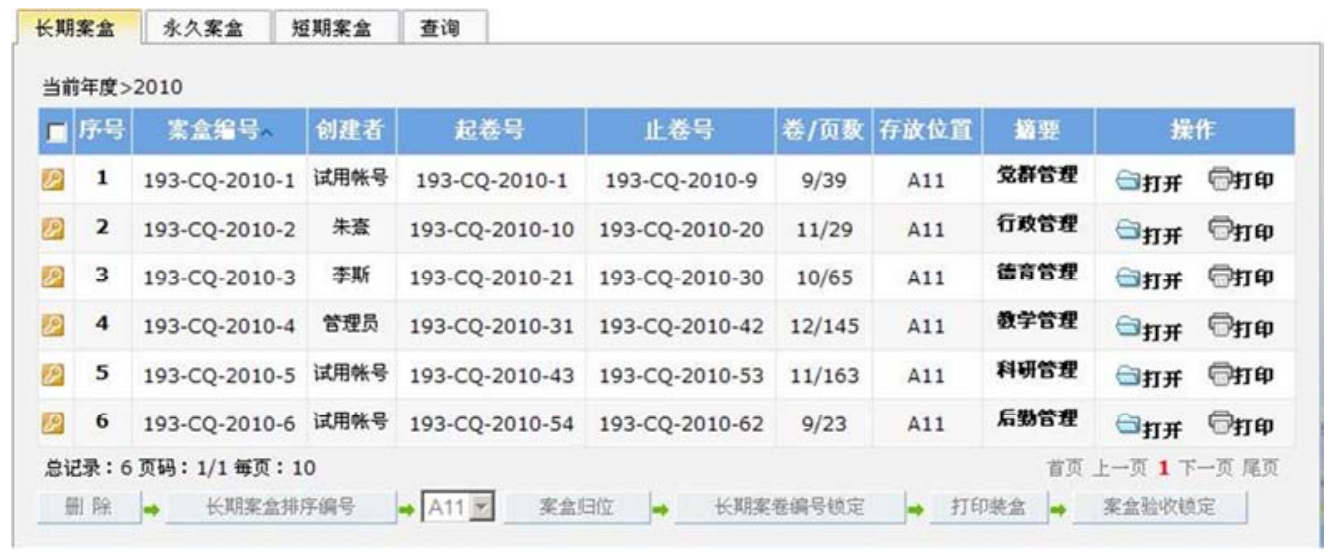

图7 案盒管理。

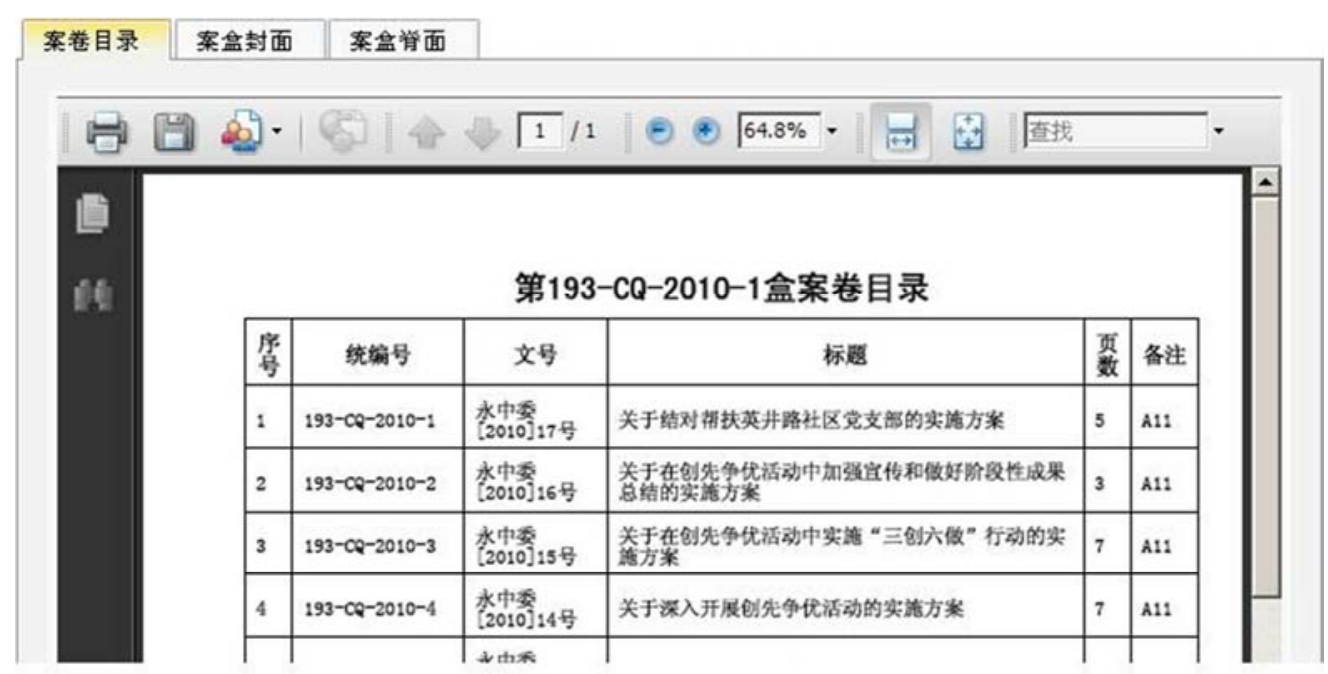

图8 案盒卷内目录打印。

\subsection{5. 专题档案模块}

案卷除能实现按常规分类归档以外, 还可实现将案卷 多次逻辑组档, 以便形成需要的专题档案, 专题档案的分 类独立于常规分类, 更为灵活, 以满足各类专项检查的需 要[7]。所有的专题档案均来自现有案卷, 这样可保持数据 的统一, 避免数据的冗余。专题档案模块除实现专题案盒 的案卷建立和调整, 提供专题案盒内的案卷的逻辑抽取等 功能。供自动生成和打印专题档案封面和卷内目录, 提供 专题档案在线汶览等功能。

\subsection{6. 借阅登记模块}

档案管理信息系统专门设计有低质档案的借阅登记 模块, 满足特殊情况需要借阅纸质案卷的需要。主要实现 案卷的借还登记管理。凡借出的案卷系统案卷状态自动更 新为“借出”, 还回的案卷, 系统的案卷状态自动更新为“在
案”。通过借阅登记的记录数据, 可以从一个则面反映纸 质档案的利用情况, 也可实时反映出案卷的状态。

\section{3. 安全保密设计}

基于网络的信息管理系统在方便使用的同时也存档 案信息安全的隐患。为规避和防范档案管理信息系统来自 网络的威胁, 在设计和实现阶段都需要高度重视档案信息 的安全问题, 特做如下两方面的分析和预案 [8]:

对于因硬软件可能造成的数据丢失或破坏, 本系统主 要采取以下防范措施:

（1）建立纸质、电子档案“双套制”。正式归档的电 子档案在统一编号后需分盒打印出纸抽档案归档入室。电 子和纸质档案哪一方因故缺失, 通过另一方都可以恢复。 这是解决电子档案安全问题根本措施。 
（2）电子档案数据集中存咜，定期备份。通过加强 数据库服务器管理, 利用MS SQL Server备份功能实现, 定期备份尽可做到异地备份。

（3）制定并落实安全责任制, 签订信息安全责任书。 凡能接触到电子档案存储服务器和档案室的相关人必须 承担相应的信息安全责任。 范措施:

对于非法访问可能带来的安全隐患主要采取以下防

(1)访问控制: 系统设置了系统管理员、档案管理员、 部门用户三类角色并分别授权, 通过登录模块对用户身份 进行识别和控制, 保证其仅能访问授权部分信息。系统还 可设置访问范围, 可限定局域网或指定IP的用户访问。对 安全性要求特别高的可以选配基于硬件的登录身份认证锁。

(2) 操作记录: 所有对档案管理信息系统的操作都 通过日志文件记录。日志文件定期备份, 系统管理员可以 通过对日志文件数据的分析追溯用户的操作行为。

(3) 入侵检测: 凡用户提交的数据都设计有检测功 能。对于网络数据流中潜在的入侵、攻击以及滥用等方面 进行自动检测。如设置了专门的SQL注入检测程序, 对用 户提交页面进行检测, 防止通过外部提交数据, 影响数据 安全。以下代码就是实现对用户提交的页面进行检测的功 能[9]。

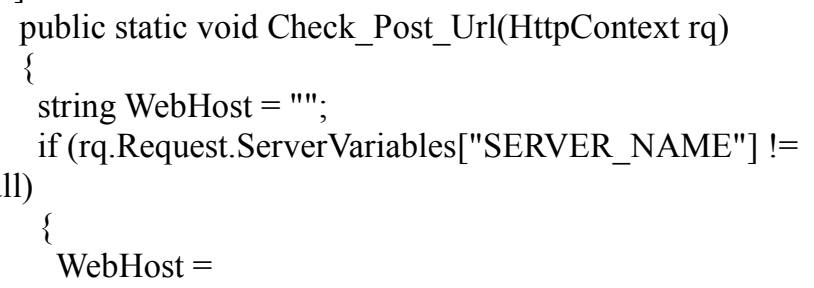

rq.Request.ServerVariables["SERVER_NAME"].ToString( )

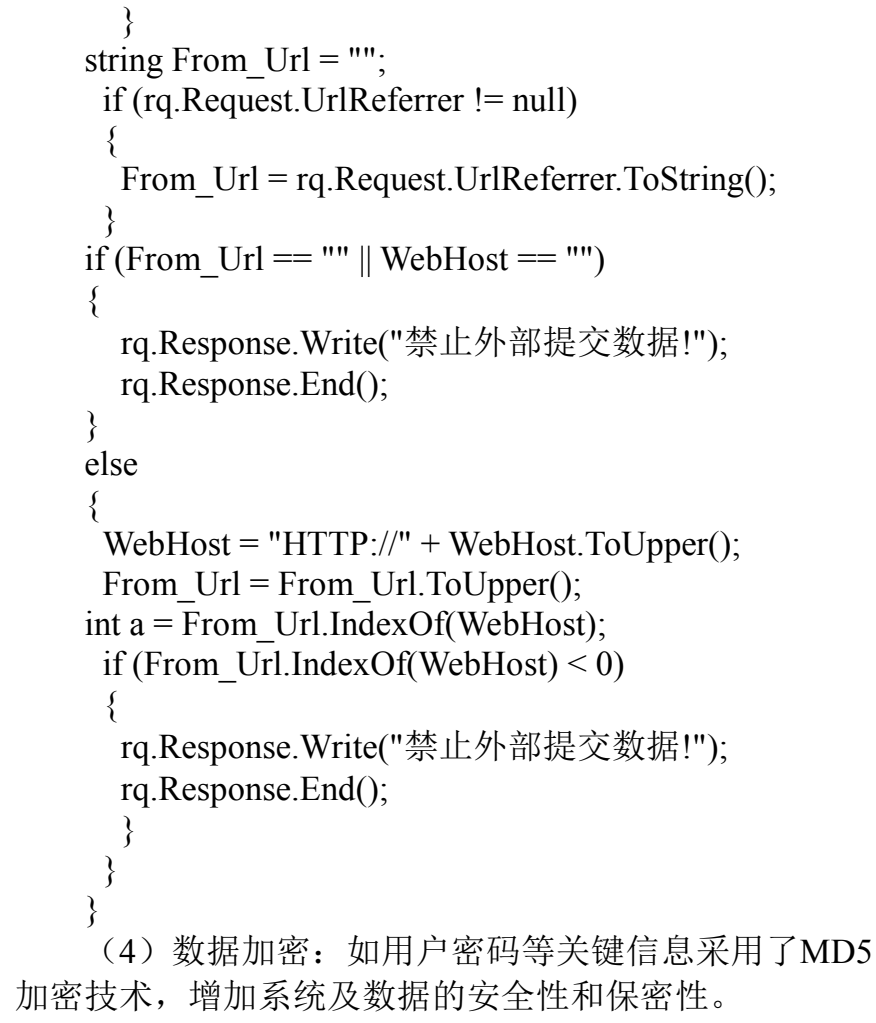

（4）数据加密：如用户密码等关键信息采用了MD5 加密技术, 增加系统及数据的安全性和保密性。

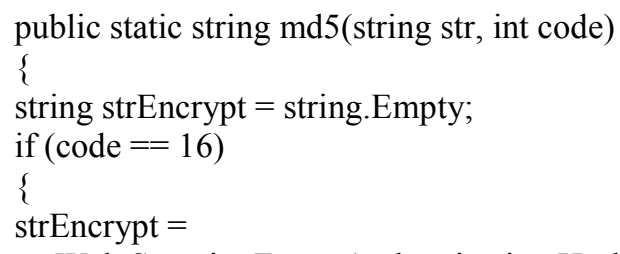

System.Web.Security.FormsAuthentication.HashPasswordFo rStoringInConfigFile(str, "MD5").Substring $(8,16)$;

\}

if $($ code $==32)$

\{

strEncrypt $=$

System.Web.Security.FormsAuthentication.HashPasswordFo rStoringInConfigFile(str, "MD5");

\}

return strEncrypt;

\}

\section{4. 业务流程设计}

\subsection{1. 划分对象层次、调整管理重心}

档案管理对象分案卷、案盒、案柜、案室四个层次, 其中核心业务层是案卷。出于规范管理的需要将以前以案 盒为单位立卷的方式改成以案卷为单位的方式立卷。以案 卷为单位进行绞边封装, 实行一件一卷, 一卷一号。案盒 是相关案卷的容器, 为后期使用方便, 案盒里的案卷建议 不封装。案柜必需统一按一定规则编号。案柜编号在系统 中纳入字典管理, 方便案盒归位。案盒实际归位要与系统 中的虚拟归位保持一致。

\subsection{2. 明确业务范围、理顺业务流程}

学校系统管理员对系统进行相应配置并对案卷类进 行设置后, 档案管理系统业务流程可分为实时立卷、集中 归盒、统一编号、打印装盒、送验归档、借阅利用六个阶 段[9]。

（1）实时立卷阶段: 由部门相关工作人员登录后, 在系统[案卷管理]模块中, 根据学校归档目录要求将本部 门产生的电子文档分管理期限、类别、年度实时上传到系 统, 对该电子文档的形式特征和内容特征进行在线著录。 对没有电子文档但使用率又较高的纸质文档通过扫描等 方式转换成电子文件上传。系统支持常见的doc、xls、ppt、 pdf、txt文书文档上传。为实现在线全文查阅，根据档案 管理对电子文档的规范要求, 需要将以上类型的文书类电 子文档统一转换成pdf格式。系统支持转换后上传、上传 后系统自动转换和上传后管理员统一转换三种方式。支持 声音文件、视频文件上传和在线视听。实时立卷要求著录 规范, 如标题、检索词应精简且能准确体现案卷主要内容, 便于以后检索。有电子文档的, 页数由系统自动识别, 无 电子文档的页数需要手动输入。默认情况下, 用户具有新 建案卷、编辑或删除自己建立的未审核编号的案卷。对已 验收入室的案卷有专题归档和查阅权限。

（2）集中归盒阶段：根据档案管理的统一安排, 由 各处室相关人员在期末或年末集中时间在系统[案盒管理] 模块中建立案盒信息, 在 [案卷管理]模块中清理或调整本 部门上传不合适的案卷, 并根据案卷分类要求, 结合案盒 
的容量对的案卷进行虚拟预归盒处理, 为下一阶段学校档 案管理员统一编号做准备。案卷可重复归盒, 但只保留最 后一次归盒。默认情况下, 用户具有建立案盒并预归盒以 及专题归盒的权限。对已验收入室的案盒只具有浏览打印 权限。

（3）统一编号阶段：由学校档案管理员集中时间在 [案盒管理]模块中, 对各处室预归盒情况进行检查, 删除 空案盒, 拆除没必要归档的案卷, 调整案盒顺序, 设定案 盒存放位置, 然后进行全校拉通统一编号。编号由系统根 据设定规则自动完成。分年（度）按期限按“全宗号一保 管期一年度十年度序号”规则拉通编号，并在案卷对应的 电子文档的右上角增加编号标识, 供下一阶段各处室打印。

（4）打印装盒阶段: 各处室人员在系统[案盒管理] 模块中打印出带编号的纸质案卷, 已有纸质案卷的打印出 标识或封面页; 纸质案卷由案卷属性和案卷两部分组成, 将纸质案卷分卷进行绞边处理, 根据系统中的预归盒顺序 装入纸质案盒; 打印出系统自动生成的卷内目录,打印出 案盒封面、案盒脊面。并粘贴到相应位置, 完成案卷装盒。

（5）送验归档阶段：由各处室档案管理人员将本部 门的案盒交学校档案室。学校档案管理员在系统[案盒管 理]模块中根据案盒相关信息进行逐一验收, 保证帐 (系 统登记的案卷和案盒) 和物 (纸质的案卷和卷盒) 一致。 对验收合格的案盒按预先设定的位置准确入室归位, 形成 年度档案，供下一阶段借阅利用。

（6）借阅利用阶段: 由于归档入室的纸质案卷与管 理系统中的电子案卷是一一对应、互为备份的, 所以各处 室可以对本部门上传的案卷通过网络实时在线查阅、打印。 也可根据迎检等需要建立专题档案, 重新对案卷进行多次 重组虚拟归档。不分保管期, 同一卷可同时归到不同的专 题类别; 专题归档直接在正式档案中提取。可自动生成专 题类别的案盒封面和案卷目录。专题档案只是逻辑上对案 卷重新分类, 增加、删除等调整操作不影响正式档案。

\section{3. 结论}

中小学校纸质档案与电子档案双套制、网络化管理是 学校档案管理的发展方向。纸质档案与电子档案一体化管 理需要整体设计解决方案。主要包括架构与技术设计、需 求与功能设计、安全保密设计、业务流程设计以及管理规 范设计。档案管理信息系统是实现纸质档案和电子档案一 体化管理的平台。档案管理信息系统的开发需要据需论证、 立项研究, 量身定制才能与档案管理业务流程相适应。只 有考虑共性需求, 兼顾个性需求, 满足变化需要的档案管 理信息系统才具有较长的软件生命周期。档案管理系统的 开发需要遵从国家标准和规范, 这样才会有利于后期开发
和利用。档案管理系统的开发需要从管理到技术两层面加 强数据安全设计, 确保档案数据的安全。档案管理信息系 统宜采用 $\mathrm{B} / \mathrm{S}$ 结构, 通过访问规则约束权限。档案管理信 息系统的实现层面宜按数据层, 业务层, 表示层三层架构 开发, 这样能使架构更清晰, 提高代码重用率, 减少代码 编写复杂度, 便于日后开发和维护。档案信息化是一个系 统工程, 需要多个部门密切配合、各环节环环相扣, 需要 与之配套的培训和管理 [10]。为此学校需要建立由校长分 管、办公室统筹、档案室具体负责、各部门负责人参与的 管理队。建立系统管理员、学校档案管理员、各部门档案 管理员组成的执行团队。需要组织专门培训, 使档案管理 人员熟悉档案管理流程, 明确档案规范, 掌握相关技术。 需要建立学校档案立卷、审核、保管、借阅等相关制度; 学校分管领导要适时协调和督促, 保证档案管理按制度规 范运行。只有这样才能使档案管理工作衔接有序,顺利推 动。

\section{参考文献}

[1] 中华人民共和国国家质量监督检验检疫总局、中国国家标 准化管理委员会.GB/T 18894-2016电子文件归档与电子档 案管理规范[S].北京.2016年。

[2] 中华人民共和国国家档案局.DA/T31-2017纸质档案数字化 规范[S].北京.2017年。

[3] 朱爱红,余冬梅,张聚礼. 基于 $\mathrm{B} / \mathrm{S}$ 软件体系结构的研究 [J]. 计 算机工程与设计,2005(05)。

[4] 王秋霞,徐忠勇.档案信息数据库建设原则研究 [J].档案学研 究, 1998(02):51-52+47。

[5] 李云全,许长有.档案信息管理系统权限管理设计[J].焦作师 范高等专科学校学报,2011,27(03):72-74+86。

[6] 中华人民共和国国家档案局.DA/T18-1999档案著录规则[S]. 北京.1999年。

[7] 江歆楠.电子文件归档管理研究 [J]. 黑龙江档案, 2015 (6)。

[8] 鲁玉芳.档案信息化建设的安全问题与对策 [J].网络安全技 术与应用,2018(01):80+82。

[9] 郑雷雷.B/S架构软件的安全性测试研究[A].中国安防展览 网.大数据环境下安防系统安全等级保护研讨会论文集 $[\mathrm{C}]$. 中国安防展览网:,2018:5。

[10] 继卫.档案信息化 [J].档案天地,2018(04):1。 